\title{
Phonon-assisted Andreev reflection in a hybrid junction based on a quantum dot
}

\author{
Kacper Bocian ${ }^{\mathrm{a}}$ and Wojciech Rudziński \\ Faculty of Physics, Adam Mickiewicz University, ul. Umultowska 85, 61-614 Poznań, Poland
}

Received 27 December 2014

Published online 18 February 2015

(C) The Author(s) 2015. This article is published with open access at Springerlink.com

\begin{abstract}
Spin-dependent tunneling through a quantum dot coupled to one ferromagnetic and one superconducting electrodes is investigated in the Andreev reflection (AR) regime occurring in the presence of the on-dot electron-phonon interactions. Current-voltage characteristics of the system are evaluated within the nonequilibrium Green function technique. Features of the AR current due to interplay between the electron-phonon interactions, intradot Coulomb correlations and the polarization of the ferromagnetic electrode are analyzed in both linear and nonlinear transport regimes. It is shown that for the case of electron-hole symmetry, the phonon resonances may appear on both sides of the main elastic resonances in spectral function. A phonon-induced renormalization of the Andreev transmission levels is found and splitting of the phonon side bands for nonvanishing intradot Coulomb repulsion is observed. It is shown that linear conductance exhibits the polaron shift and suppression in the presence of the polaron transmission. In nonequilibrium situation the mechanism of the negative differential conductance (NDC) in the presence of competing magnetic electrode polarisation and the Coulomb correlations on the dot is analyzed. An influence of the polaron transmission through the Andreev intradot states on the NDC oscillations is also discussed.
\end{abstract}

\section{Introduction}

Phonon-assisted Andreev reflection (AR) [1] in hybrid mesoscopic devices based on quantum dots (QDs) or molecules attached to external normal metallic $(\mathrm{N})$, ferromagnetic (F) and superconducting (S) electrodes has been investigated recently in a number of theoretical papers [2-7]. On experimental side, up to now only the point-contact studies on effects due to phonons in transport across the normal-metal/superconductor interface have been reported (see e.g. [8]). The Andreev reflection measurements in point-contact systems revealed nonlinearities in the current-voltage characteristics containing information on the energy of the electron-phonon interaction. Theoretical works on Andreev transmission through QDs attached to N, F and S leads predict appearance of polaron-induced resonances in the conductance characteristics. It is found that for the F-QD-S system with vanishing intradot Coulomb correlations [2], the phonon peaks in the AR conductance are rather weak. By contrast, in reference [3] it is shown that the coherent intradot spin rotation in the F-QD-S hybrid tunneling junction may enhance the AR current in the nonequilibrium situation. In turn, investigation on phonon-assisted Andreev and

\footnotetext{
a e-mail: bocian@amu.edu.pl
}

normal tunnelings through N-QD-S junction with vanishing effective intradot Coulomb repulsion revealed that the phonon resonances observed in the Andreev conductance are robust against the thermal disturbance. The features of heat generation in Andreev transmission through the N-QD-S system, have been also the subject of theoretical analysis [6]. The discussion implied that the currentinduced heat generation is influenced significantly by the QD-S coupling strength and that the intradot phonon mode gives rise to additional steps in the non-equilibrium heat generation characteristics. Finally, the features of AR spectral function for tunneling through the interacting QD attached to $\mathrm{F}$ and $\mathrm{S}$ electrodes have been analyzed in reference [7]. It has been found that in the case of a F-QD coupling much weaker than the QD-S one, the Andreev bound states may be reproduced in phonon satellites. A renormalization of the AR bound sates has been also predicted to occur for QD interacting with phonons.

In the present paper we analyze phonon-induced effects in Andreev spin-polarized transmission through the F-QD-S hybrid system based on a quantum dot with arbitrary effective Coulomb correlations. The features of the relevant current-voltage characteristics are investigated for interplay between phonon-electron interactions, effective intradot Coulomb correlations, polarization of the $\mathrm{F}$ lead and the dot-electrode coupling strengths. 
The discussion of reference [7] on such effects as the renormalization of the Andreev energy levels due to phonons or the splitting of the phonon side bands in spectral function induced by intradot Coulomb repulsion is extended for QD symmetrically coupled to the F and S leads. The analysis of the equilibrium situation comprises also investigation on the polaron shift of the linear conductance in the Andreev reflection regime as well as the influence of competing $\mathrm{F}$ lead polarization and the Franck-Condon blockade on suppression of the linear conductance. New features have been predicted to occur in nonequilibrium situation for which the map dependencies of differential conductance have been calculated versus a wide range of the bias voltage, effective on-dot Coulomb repulsion parameter $\left(U^{\prime}\right)$ as well as the $\mathrm{F}$ lead polarization. In particular, the effects due to overlap of the phonon and main resonances for arbitrary Coulomb correlations are observed, the condition of the $U^{\prime}$-dependent enhancement of the conductance is determined in situation when electronphonon interactions tend to suppress the AR transmission. We also discuss another mechanism of occurrence of negative differential conductance (NDC) in hybrid tunneling devices, which is different from the one described previously for the Andreev tunneling through a double quantum dot attached to $\mathrm{F}$ and $\mathrm{S}$ external leads [9]. Consequently, the effect of oscillations of NDC is found to occur due to the phonon-assisted transmission in the AR regime and it is studied in the context of tunneling processes between the dot and differently polarized $\mathrm{F}$ electrode.

In order to calculate transport $\mathrm{AR}$ characteristics in the considered system we use the nonequilibrium Green function technique in the $4 \times 4 \mathrm{Nambu}$ representation. All the Green function correlators are derived by means of the equation of motion method in the Hubbard I approximation. Moreover, taking into account the results of previous discussion on different approximations used to evaluate the Green functions for the dot interacting with phonons (for details see e.g. [10-12]), we consider the effects due to both the electron and hole transmission. Thus at zero temperature and for the electron-hole symmetry our approach results in appearance of the phonon emission satellites on both sides of the main elastic peaks in spectral function. It is also shown that due to the applied approach the polaron shift of linear conductance occurs without the appearance of the phonon satellites.

The paper is organized as follows. In Section 2 we describe model of the system and method. Numerical results are discussed in Section 3, while summary and general conclusions are in Section 4.

\section{Model and method}

We consider a single-level QD coupled via tunnel barriers to one ferromagnetic and one superconducting leads. Such a hybrid junction can be described by Hamiltonian of the form

$$
H=H_{f}+H_{s}+H_{p h}+H_{d}+H_{t}
$$

The term $H_{f}$ describes the ferromagnetic electrode in the non-interacting, quasi-particle approximation,

$$
H_{f}=\sum_{k, \sigma} \varepsilon_{k \sigma} f_{k \sigma}^{+} f_{k \sigma}
$$

where $\varepsilon_{k \sigma}$ is the single-electron energy for wavevector $k$ and spin $\sigma(\sigma=\uparrow, \downarrow)$ in the ferromagnetic electrode, whereas $f_{k \sigma}^{+}$and $f_{k \sigma}$ are the corresponding creation and annihilation operators. The second term is the BCS Hamiltonian of the superconducting lead,

$$
H_{s}=\sum_{p, \sigma} \varepsilon_{p} s_{p \sigma}^{+} s_{p \sigma}+\sum_{p}\left(\Delta^{*} s_{p \uparrow}^{+} s_{-p \downarrow}^{+}+\Delta s_{-p \downarrow} s_{p \uparrow}\right),
$$

with $\varepsilon_{p}$ denoting the single-particle spectrum in the corresponding normal-metal phase, and $\Delta$ being the parameter describing the superconducting energy gap. The third term is the phonon Hamiltonian:

$$
H_{p h}=\omega_{0} b^{+} b
$$

where $\omega_{0}$ is the vibrational frequency of the phonon mode and $b^{+}(b)$ is the phonon creation (annihilation) operator. The quantum dot is described by:

$$
H_{d}=\sum_{\sigma}\left[\varepsilon_{d}+\lambda\left(b+b^{+}\right)\right] d_{\sigma}^{+} d_{\sigma}+U d_{\uparrow}^{+} d_{\uparrow} d_{\downarrow}^{+} d_{\downarrow}
$$

where $\varepsilon_{d}$ denotes the energy of the discrete level, $U$ is the electron correlation parameter, the parameter $\lambda$ describes strength of the electron-phonon coupling, $d_{\sigma}^{+}$and $d_{\sigma}$ is the creation and annihilation operator, respectively, for electrons with spin orientation $\sigma=\uparrow(\downarrow)$. Finally, the tunnelling term in equation (1) takes the form

$$
H_{t}=\sum_{k, \sigma} T_{k \sigma}^{f} f_{k \sigma}^{+} d_{\sigma}+\sum_{p, \sigma} T_{p \sigma}^{s} s_{p \sigma}^{+} d_{\sigma}+\text { h.c. }
$$

where $T_{k \sigma}^{f}\left(T_{p \sigma}^{s}\right)$ is the tunneling amplitude between the ferromagnetic (superconducting) lead and the QD, and h.c. stands for the hermitian conjugate terms.

In the following, to eliminate the linear coupling terms in the Hamiltonian $H_{d}$, we make use of the following LangFirsov-type unitary transformation [13]:

$$
\tilde{H}=e^{S} H e^{-S}, \quad S=\frac{\lambda}{\omega_{0}} \sum_{\sigma} d_{\sigma}^{+} d_{\sigma}\left(b^{+}-b\right),
$$

which implies that the fermion and boson operators become

$$
\begin{aligned}
\tilde{d}_{\sigma} & =d_{\sigma} X \\
\tilde{d}_{\sigma}^{+} & =d_{\sigma}^{+} X^{+}, \\
\tilde{b} & =b-\sum_{\sigma} \frac{\lambda}{\omega_{0}} d_{\sigma}^{+} d_{\sigma}, \\
\tilde{b}^{+} & =b^{+}-\sum_{\sigma} \frac{\lambda}{\omega_{0}} d_{\sigma}^{+} d_{\sigma},
\end{aligned}
$$


where $X$ is the phonon operator, $X=\exp \left[-\left(\lambda / \omega_{0}\right)\left(b^{+}-\right.\right.$ $b)]$. Thus, the Hamiltonian (1) may be now written in the following form

$$
\tilde{H}=H_{f}+H_{s}+H_{p h}+\tilde{H}_{d}+\tilde{H}_{t},
$$

where

$$
\tilde{H}_{d}=\sum_{\sigma} \varepsilon_{d}^{\prime} d_{\sigma}^{+} d_{\sigma}+U^{\prime} d_{\uparrow}^{+} d_{\uparrow} d_{\downarrow}^{+} d_{\downarrow}
$$

and

$$
\tilde{H}_{t}=\sum_{k, \sigma} T_{k \sigma}^{\prime f} f_{k \sigma}^{+} d_{\sigma}+\sum_{p, \sigma} T_{p \sigma}^{\prime s} s_{p \sigma}^{+} d_{\sigma}+\text { h.c. }
$$

with the renormalized dot energy level, $\varepsilon_{d}^{\prime}=\varepsilon_{d}-\lambda^{2} / \omega_{0}$, effective charging energy $U^{\prime}=U-2 \lambda^{2} / \omega_{0}$, and with tunneling matrix elements given by $T_{k, p \sigma}^{\prime f, s}=T_{k, p \sigma}^{f, s} X$. Assuming that $T_{k, p \sigma}^{f, s} \ll \lambda$, which is valid provided that the tunneling amplitudes does not depend on the polaron position, one may adopt the approximation developed for the independent boson model [11,13-15] by replacing the phonon operator $X$ in equation (14) with its expectation value, $\langle X\rangle=\exp \left[-\left(\lambda / \omega_{0}\right)^{2}\left(N_{p h}+1 / 2\right)\right]$, where $N_{p h}$ is the Bose distribution, $N_{p h}=1 /\left[\exp \left(\beta \omega_{0}\right)-1\right]$, and $\beta=1 / k_{B} T$. Thus, exponential suppression of the tunneling amplitudes in the tunneling Hamiltonian (12) leads to the charge conserving $\lambda$-dependent Franck-Condon blockade of tunneling processes between the dot and the F electrode.

To calculate current-voltage characteristics in the non-equilibrium situation we apply the nonequilibrium Green-function technique in the $4 \times 4$ Nambu representation [16]. Accordingly, by using the Nambu spinor $\boldsymbol{\Psi}(t)=\left(d_{\uparrow}^{\dagger}, d_{\downarrow}, d_{\downarrow}^{\dagger}, d_{\uparrow}\right)^{\dagger}$ we introduce the causal (timeordered) Green function of the $\operatorname{dot} \mathbf{G}\left(t, t^{\prime}\right)=-i\left\langle T_{t}[\mathbf{\Psi}(t) \otimes\right.$ $\left.\left.\boldsymbol{\Psi}^{\dagger}\left(t^{\prime}\right)\right]_{\eta}\right\rangle$, where the symbol $T_{t}$ is time-ordering operator, $\eta= \pm 1$ (with "+" and "-" sign corresponding to bosons and fermions, respectively), whereas $\otimes$ denotes the outer product $(\mathbf{a} \otimes \mathbf{b})_{i j}=a_{i} b_{j}$. Thus, the corresponding retarded Green function takes the form $\mathbf{G}^{r}\left(t, t^{\prime}\right)=$ $-i \theta\left(t-t^{\prime}\right)\left\langle\left[\boldsymbol{\Psi}(t) \otimes \boldsymbol{\Psi}^{\dagger}\left(t^{\prime}\right)\right]_{\eta}\right\rangle$, whereas the advanced one reads $\mathbf{G}^{a}\left(t, t^{\prime}\right)=i \theta\left(t^{\prime}-t\right)\left\langle\left[\mathbf{\Psi}(t) \otimes \boldsymbol{\Psi}^{\dagger}\left(t^{\prime}\right)\right]_{\eta}\right\rangle$, with $\theta$ being the Heaviside step function. We also introduce the lesser and greater correlators defined as: $\mathbf{G}^{<}\left(t, t^{\prime}\right)=i\left\langle\mathbf{\Psi}^{\dagger}\left(t^{\prime}\right) \otimes\right.$ $\boldsymbol{\Psi}(t)\rangle$ and $\mathbf{G}^{>}\left(t, t^{\prime}\right)=-i\left\langle\boldsymbol{\Psi}(t) \otimes \boldsymbol{\Psi}^{\dagger}\left(t^{\prime}\right)\right\rangle$, respectively. From equations (7)-(9) it follows that the canonical transformation gives rise to the Nambu spinor of the form $\tilde{\boldsymbol{\Psi}}(t)=\left(\tilde{d}_{\uparrow}^{\dagger}, \tilde{d}_{\downarrow}, \tilde{d}_{\downarrow}^{\dagger}, \tilde{d}_{\uparrow}\right)^{\dagger}=\left(d_{\uparrow}^{\dagger} X^{\dagger}, d_{\downarrow} X, d_{\downarrow}^{\dagger} X^{\dagger}, d_{\uparrow} X\right)^{\dagger}=$ $\left(d_{\uparrow}^{\dagger}, d_{\downarrow}, d_{\downarrow}^{\dagger}, d_{\uparrow}\right)^{\dagger} \circ\left(X^{\dagger}, X, X^{\dagger}, X\right)^{\dagger}=\boldsymbol{\Psi}(t) \circ \boldsymbol{\Omega}(t)$, where symbol $\circ$ stands for the Hadamard product $(\mathbf{A} \circ \mathbf{B})_{i j}=$ $A_{i j} B_{i j}$. After some analytical manipulation (see Appendix for details) we arrive at Fourier transform for the retarded and lesser Green functions

$$
\begin{aligned}
\mathbf{G}^{r}(\omega)= & \sum_{n=-\infty}^{\infty} L_{n}\left[\tilde{\mathbf{G}}^{\mathbf{r}}\left(\omega-n \omega_{0}\right)+\frac{1}{2} \tilde{\mathbf{G}}^{<}\left(\omega-n \omega_{0}\right)\right. \\
& \left.-\frac{1}{2} \tilde{\mathbf{G}}^{<}\left(\omega+n \omega_{0}\right)\right] \circ \mathbf{M}_{n} \\
\mathbf{G}^{<}(\omega)= & \sum_{n=-\infty}^{\infty} L_{n} \tilde{\mathbf{G}}^{<}\left(\omega+n \omega_{0}\right) \circ \mathbf{M}_{n}
\end{aligned}
$$

with

$$
\mathbf{M}_{n}=\left(\begin{array}{cccc}
1 & (-1)^{n} & 1 & (-1)^{n} \\
(-1)^{n} & 1 & (-1)^{n} & 1 \\
1 & (-1)^{n} & 1 & (-1)^{n} \\
(-1)^{n} & 1 & (-1)^{n} & 1
\end{array}\right)
$$

and with $\tilde{\mathbf{G}}^{r}(\omega)$ and $\tilde{\mathbf{G}}^{<}(\omega)$ being the so-called dressed correlators obtained as a result of the Lang-Firsov transformation (7). The symbol $L_{n}$ stands for the FranckCondon factor which is given by:

$$
L_{n}=e^{-\left(\frac{\lambda}{\omega_{0}}\right)^{2}}\left(\frac{\lambda}{\omega_{0}}\right)^{2 n}\left(\frac{1}{n !}\right)
$$

for $T=0$ and

$L_{n}=e^{-\left(\frac{\lambda}{\omega_{0}}\right)^{2}\left(2 N_{p h}+1\right)} e^{\frac{n \omega_{0} \beta}{2}} I_{n}\left[2\left(\frac{\lambda}{\omega_{0}}\right)^{2} \sqrt{N_{p h}\left(N_{p h}+1\right)}\right]$

for $T>0$, where $I_{n}$ is the $n$th Bessel function of the first kind.

The explicite expression for the dressed retarded Green functions can be obtained by means of the equation of motion method. To obtain the $\tilde{\mathbf{G}}^{r}(\omega)$ correlator it is convenient to derive first the Fourier transforms of the causal Green functions defined as $\left\langle\left\langle d_{\sigma} \mid d_{\sigma^{\prime}}^{+}\right\rangle\right\rangle_{\tilde{H}},\left\langle\left\langle d_{\sigma} \mid d_{\sigma^{\prime}}\right\rangle\right\rangle_{\tilde{H}}$, $\left\langle\left\langle d_{\sigma}^{+} \mid d_{\sigma^{\prime}}\right\rangle\right\rangle_{\tilde{H}}$ and $\left\langle\left\langle d_{\sigma}^{+} \mid d_{\sigma^{\prime}}^{+}\right\rangle\right\rangle_{\tilde{H}}$, where $\langle\langle\mid\rangle\rangle_{\tilde{H}}$ denotes the Zubarev notation [17], and where the subscript $\tilde{H}$ indicates that a given correlator is governed by the transformed Hamiltonian (12). In particular, the equation of motion for the function $\left\langle\left\langle d_{\sigma} \mid d_{\sigma^{\prime}}^{+}\right\rangle\right\rangle_{\tilde{H}}$ reads:

$$
\begin{aligned}
\left(\varepsilon-\varepsilon_{d \sigma}^{\prime}\right)\left\langle\left\langle d_{\sigma} \mid d_{\sigma^{\prime}}^{\dagger}\right\rangle\right\rangle_{\tilde{H}}= & \delta_{\sigma \sigma^{\prime}}+U^{\prime}\left\langle\left\langle d_{\sigma} d_{-\sigma}^{+} d_{-\sigma} \mid d_{\sigma^{\prime}}^{+}\right\rangle\right\rangle_{\tilde{H}} \\
& +\sum_{k} T_{k \sigma}^{\prime f \star}\left\langle\left\langle f_{k \sigma} \mid d_{\sigma^{\prime}}^{\dagger}\right\rangle\right\rangle_{\tilde{H}} \\
& +\sum_{p} T_{p \sigma}^{\prime} s \star\left\langle\left\langle s_{p \sigma} \mid d_{\sigma^{\prime}}^{\dagger}\right\rangle\right\rangle_{\tilde{H}},
\end{aligned}
$$


where

$$
\begin{gathered}
\left(\varepsilon-\varepsilon_{k \sigma}\right)\left\langle\left\langle f_{k \sigma} \mid d_{\sigma^{\prime}}^{\dagger}\right\rangle\right\rangle_{\tilde{H}}=T_{k \sigma}^{\prime f}\left\langle\left\langle d_{\sigma} \mid d_{\sigma^{\prime}}^{\dagger}\right\rangle\right\rangle_{\tilde{H}} \\
{\left[\left(\varepsilon+\varepsilon_{p-\sigma}\right)\left(\varepsilon-\varepsilon_{p \sigma}\right)-|\Delta|^{2}\right]\left\langle\left\langle s_{p \sigma} \mid d_{\sigma^{\prime}}^{\dagger}\right\rangle\right\rangle_{\tilde{H}}} \\
=-\sigma \Delta^{\star} T_{p-\sigma}^{s \star}\left\langle\left\langle d_{-\sigma}^{\dagger} \mid d_{\sigma^{\prime}}^{\dagger}\right\rangle\right\rangle_{\tilde{H}} \\
+T_{p \sigma}^{\prime s}\left(\varepsilon+\varepsilon_{p-\sigma}\right)\left\langle\left\langle d_{\sigma} \mid d_{\sigma^{\prime}}^{\dagger}\right\rangle\right\rangle_{\tilde{H}} \\
\left(\varepsilon-\varepsilon_{d \sigma}^{\prime}-U^{\prime}\right)\left\langle\left\langle d_{\sigma} d_{-\sigma}^{+} d_{-\sigma} \mid d_{\sigma^{\prime}}^{+}\right\rangle\right\rangle_{\tilde{H}}=\delta_{\sigma \sigma^{\prime}}\left\langle d_{-\sigma}^{\dagger} d_{-\sigma}\right\rangle \\
+\left\langle d_{-\sigma}^{\dagger} d_{-\sigma}\right\rangle \sum_{k} T_{k \sigma}^{\prime f \star}\left\langle\left\langle f_{k \sigma} \mid d_{\sigma^{\prime}}^{\dagger}\right\rangle\right\rangle_{\tilde{H}} \\
+\left\langle d_{-\sigma}^{\dagger} d_{-\sigma}\right\rangle \sum_{p} T_{p \sigma}^{s \star}\left\langle\left\langle s_{p \sigma} \mid d_{\sigma^{\prime}}^{\dagger}\right\rangle\right\rangle_{\tilde{H}}
\end{gathered}
$$

In obtaining the equation of motion for the higherorder Green function in equation (23) we have assumed that the AR transmission occurs without spinflip processes on the dot, $\left\langle d_{-\sigma}^{\dagger} d_{\sigma}\right\rangle=0$, as well as without assistance of Cooper pairs on the dot, $\left\langle d_{\sigma} d_{-\sigma}\right\rangle=0$. We have also neglected correlations involving lead electrons and thus the following decoupling scheme (the so-called Hubbard I approximation) was applied $\left\langle\left\langle\xi d_{-\sigma}^{\dagger} d_{-\sigma} \mid d_{\sigma^{\prime}}^{\dagger}\right\rangle\right\rangle_{\tilde{H}} \rightarrow\left\langle d_{-\sigma}^{\dagger} d_{-\sigma}\right\rangle\left\langle\left\langle\xi \mid d_{\sigma^{\prime}}^{\dagger}\right\rangle\right\rangle_{\tilde{H}}$ with $\xi=$ $f_{k \sigma}, s_{p \sigma}$. These approximations allow to close the set of equations (20)-(23). In the same manner one may write down appropriate equations of motion for the Green functions $\left\langle\left\langle d_{\sigma} \mid d_{\sigma^{\prime}}\right\rangle\right\rangle_{\tilde{H}},\left\langle\left\langle d_{\sigma}^{+} \mid d_{\sigma^{\prime}}\right\rangle\right\rangle_{\tilde{H}}$ and $\left\langle\left\langle d_{\sigma}^{+} \mid d_{\sigma^{\prime}}^{+}\right\rangle\right\rangle_{\tilde{H}}$. Finally one finds

$$
\begin{aligned}
& \left\langle\left\langle d_{\sigma} \mid d_{\sigma^{\prime}}^{\dagger}\right\rangle\right\rangle_{\tilde{H}}=\delta_{\sigma \sigma^{\prime}} \frac{A_{-\sigma}^{+} B_{\sigma}^{-}}{A_{-\sigma}^{+} A_{\sigma}^{-}-B_{-\sigma}^{+} B_{\sigma}^{-} C^{2}}, \\
& \left\langle\left\langle d_{\sigma}^{\dagger} \mid d_{\sigma^{\prime}}\right\rangle\right\rangle_{\tilde{H}}=\delta_{\sigma \sigma^{\prime}} \frac{A_{-\sigma}^{-} B_{\sigma}^{+}}{A_{\sigma}^{+} A_{-\sigma}^{-}-B_{\sigma}^{+} B_{-\sigma}^{-} C^{2}}, \\
& \left\langle\left\langle d_{\sigma}^{\dagger} \mid d_{\sigma^{\prime}}^{\dagger}\right\rangle\right\rangle_{\tilde{H}}=-\sigma \delta_{-\sigma \sigma^{\prime}} \frac{B_{\sigma}^{+} B_{-\sigma}^{-} C}{A_{\sigma}^{+} A_{-\sigma}^{-}-B_{\sigma}^{+} B_{-\sigma}^{-} C^{2}}, \\
& \left\langle\left\langle d_{\sigma} \mid d_{\sigma^{\prime}}\right\rangle\right\rangle_{\tilde{H}}=\sigma \delta_{-\sigma \sigma^{\prime}} \frac{B_{-\sigma}^{+} B_{\sigma}^{-} C}{A_{-\sigma}^{+} A_{\sigma}^{-}-B_{-\sigma}^{+} B_{\sigma}^{-} C^{2}},
\end{aligned}
$$

where

$$
\begin{aligned}
A_{\sigma}^{ \pm}= & \varepsilon \pm \varepsilon_{d \sigma}^{\prime}+\frac{i}{2} e^{-\left(\lambda / \omega_{0}\right)^{2}}\left[\Gamma_{f 0}(1+\sigma P)+\Gamma_{s 0} \rho_{s}\right] \\
& \times\left(1 \mp \frac{U^{\prime}\left\langle d_{-\sigma}^{\dagger} d_{-\sigma}\right\rangle}{\varepsilon \pm \varepsilon_{d \sigma}^{\prime} \pm U^{\prime}}\right) \\
B_{\sigma}^{ \pm}= & 1 \mp \frac{U^{\prime}\left\langle d_{-\sigma}^{\dagger} d_{-\sigma}\right\rangle}{\varepsilon \pm \varepsilon_{d \sigma}^{\prime} \pm U^{\prime}} \\
C= & \frac{i}{2} e^{-\left(\lambda / \omega_{0}\right)^{2}} \Gamma_{s 0} \frac{\Delta}{\omega} \rho_{s} .
\end{aligned}
$$

The symbol $P$ in equation (28) is the spin polarization factor, the quantity $\Gamma_{f 0}\left(\Gamma_{s 0}\right)$ determines the coupling strength between the dot and the ferromagnetic (superconducting) lead, while the symbol $\rho_{s}$ denotes the BCS density of states [18]:

$$
\rho_{s}=\frac{|\omega| \theta(|\omega|-\Delta)}{\sqrt{\omega^{2}-\Delta^{2}}}+\frac{\omega \theta(\Delta-|\omega|)}{i \sqrt{\Delta^{2}-\omega^{2}}} .
$$

The lesser (greater) Green functions $\tilde{G}_{\sigma \sigma^{\prime}}^{<(>)}(\omega)$ can be found from the Keldysh equation:

$$
\tilde{\mathbf{G}}^{<(>)}(\omega)=\tilde{\mathbf{G}}^{r}(\omega) \tilde{\boldsymbol{\Sigma}}^{<(>)}(\omega) \tilde{\mathbf{G}}^{a}(\omega),
$$

where the self energies $\tilde{\boldsymbol{\Sigma}}^{<(>)}$are given by:

$$
\begin{aligned}
& \tilde{\boldsymbol{\Sigma}}^{<}(\omega)=i\left[\mathbf{f}_{f}(\omega) \tilde{\boldsymbol{\Gamma}}_{f}+\mathbf{f}_{s}(\omega) \tilde{\boldsymbol{\Gamma}}_{s}(\omega)\right], \\
& \tilde{\boldsymbol{\Sigma}}^{>}(\omega)=-i\left\{\left[\mathbf{I}-\mathbf{f}_{f}(\omega)\right] \tilde{\boldsymbol{\Gamma}}_{f}+\left[\mathbf{I}-\mathbf{f}_{s}(\omega)\right] \tilde{\boldsymbol{\Gamma}}_{s}(\omega)\right\} .
\end{aligned}
$$

The matrices $\tilde{\boldsymbol{\Gamma}}_{f}$ and $\tilde{\boldsymbol{\Gamma}}_{s}(\omega)$ in equations (33) and (34) describe coupling of the dot to the ferromagnetic and superconducting lead, respectively,

$$
\begin{gathered}
\tilde{\boldsymbol{\Gamma}}_{f}=e^{-\left(\frac{\lambda}{\omega_{0}}\right)^{2}\left(2 N_{p h}+1\right)} \boldsymbol{\Gamma}_{f}, \\
\tilde{\boldsymbol{\Gamma}}_{s}(\omega)=e^{-\left(\frac{\lambda}{\omega_{0}}\right)^{2}\left(2 N_{p h}+1\right)} \boldsymbol{\Gamma}_{s}(\omega),
\end{gathered}
$$

with

$$
\begin{gathered}
\Gamma_{f}=\Gamma_{f 0}\left(\begin{array}{cccc}
1+P & 0 & 0 & 0 \\
0 & 1-P & 0 & 0 \\
0 & 0 & 1-P & 0 \\
0 & 0 & 0 & 1+P
\end{array}\right), \\
\Gamma_{s}(\omega)=\operatorname{Re}\left(\rho_{s}\right) \Gamma_{s 0}\left(\begin{array}{cccc}
1 & -\frac{\Delta}{\omega} & 0 & 0 \\
-\frac{\Delta}{\omega} & 1 & 0 & 0 \\
0 & 0 & 1 & \frac{\Delta}{\omega} \\
0 & 0 & \frac{\Delta}{\omega} & 1
\end{array}\right),
\end{gathered}
$$

whereas the quantities $\mathbf{f}_{f}(\omega)$ and $\mathbf{f}_{s}(\omega)$ stand for the corresponding matrices of the Fermi-Dirac distribution functions expressed by:

$\mathbf{f}_{f}(\omega)=\left(\begin{array}{cccc}f(\omega-e V) & 0 & 0 & 0 \\ 0 & f(\omega+e V) & 0 & 0 \\ 0 & 0 & f(\omega-e V) & 0 \\ 0 & 0 & 0 & f(\omega+e V)\end{array}\right)$,

and

$$
\mathbf{f}_{s}(\omega)=f(\omega) \mathbf{I},
$$

with $f(\omega)=1 /[\exp (\beta \omega)+1]$

Having found the Green functions, one can calculate the current flowing through the junction using the general formula $[19,20]$,

$$
\begin{aligned}
J= & \frac{i e}{2 \hbar} \int \frac{d \omega}{2 \pi} \operatorname{Tr}\left\{\left[\boldsymbol{\Gamma}_{f}-\boldsymbol{\Gamma}_{s}(\omega)\right] \mathbf{G}^{<}(\omega)-i\left[\mathbf{f}_{f}(\omega) \boldsymbol{\Gamma}_{f}\right.\right. \\
& \left.\left.-\mathbf{f}_{s}(\omega) \boldsymbol{\Gamma}_{s}(\omega)\right] \mathbf{A}(\omega)\right\},
\end{aligned}
$$

where the quantity $\mathbf{A}(\omega)$ stands for the spectral function,

$$
\mathbf{A}(\omega)=i\left[\mathbf{G}^{>}(\omega)-\mathbf{G}^{<}(\omega)\right] .
$$




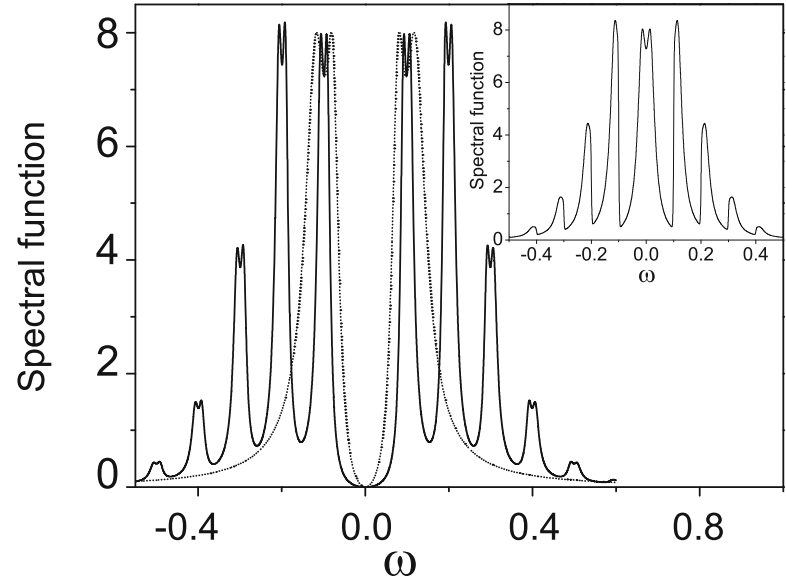

Fig. 1. AR spectral function versus energy for the electronhole symmetry, $\varepsilon_{d}^{\prime}+U^{\prime} / 2=0$. The parameters are $\lambda=0$ (dotted line), $\lambda=0.1$ (solid line), $U^{\prime}=0.2, \Gamma_{f 0}=\Gamma_{s 0}=0.1$ and $P=0.5$. The inset shows the corresponding spectral function for $\lambda=0.1$ with vanishing intradot Coulomb repulsion, $U^{\prime}=0$.

Thus, finally the AR current reads

$$
\begin{gathered}
J=\frac{i e}{2 \hbar} \int \frac{d \omega}{2 \pi} \sum_{n=-\infty}^{\infty} L_{n}\left\{\left[\tilde{\boldsymbol{\Gamma}}_{f}-\tilde{\boldsymbol{\Gamma}}_{s}(\omega)\right] \tilde{\mathbf{G}}^{<}\left(\omega+n \omega_{0}\right) \circ \mathbf{M}_{n}\right. \\
\quad+\left[\mathbf{f}_{f}(\omega) \tilde{\boldsymbol{\Gamma}}_{f}-\mathbf{f}_{s}(\omega) \tilde{\boldsymbol{\Gamma}}_{s}(\omega)\right] \\
\left.\times\left[\tilde{\mathbf{G}}^{>}\left(\omega-n \omega_{0}\right)-\tilde{\mathbf{G}}^{<}\left(\omega+n \omega_{0}\right)\right] \circ \mathbf{M}_{n}\right\}_{11+33}, \quad(43)
\end{gathered}
$$

where the index $11+33$ denotes a sum over the electron components in the Nambu space matrix.

\section{Numerical results}

Discuss features of the phonon-assisted transport characteristics at zero temperature, $T=0$, in the Andreev reflection regime for the F-QD-S system with symmetrical coupling between the dot and the leads, $\Gamma_{f 0}=\Gamma_{s 0}$, in both the linear and nonlinear response regimes. Energy is measured from the Fermi level at equilibrium, and for the AR current calculations the energy gap of the superconducting lead is assumed to be $\Delta \approx 1$. All energy-dependent parameters are given in unit $\mathrm{eV}$.

\subsection{Linear response regime, $\mathrm{V} \approx 0$}

Spectral functions for the system in equilibrium situation versus the energy $\omega$ are shown in Figure 1. When AR occurs without phonon assistance $(\lambda=0)$, then for arbitrary, non-vanishing on-dot effective Coulomb repulsion one observes two elastic resonances. Due to the assumed electron-hole symmetry these resonances are located at energies $-U^{\prime} / 2$ and $U^{\prime} / 2$ (dotted line in Fig. 1). Moreover, notice that each elastic resonance is split for AR processes. Phonons interacting with carriers residing on

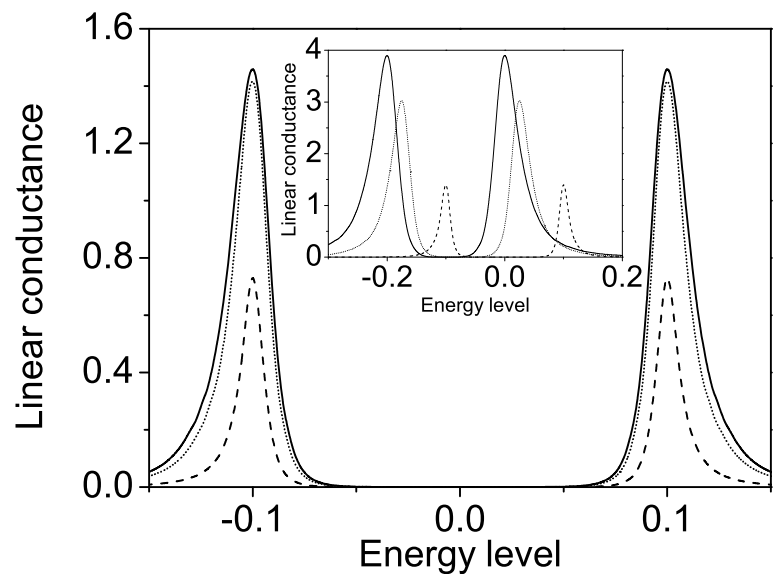

Fig. 2. AR linear conductance versus dot energy level, $\varepsilon_{d}^{\prime}$, calculated for selected values of the polarization of the $\mathrm{F}$ lead: $P=0$ (solid line), $P=0.5$ (dotted line), $P=0.9$ (dashed line). The other parameters are $U^{\prime}=0.2, \Gamma_{f 0}=\Gamma_{s 0}=0.1$ and $\lambda=0.1$. The inset shows the polaron shift for the case of $P=0.5, \lambda=0$ (solid line), $\lambda=0.05$ (dotted line) and $\lambda=0.1$ (dashed line).

the dot give rise to side bands located on both sides of the main resonances at positions corresponding to the multiple of the phonon energy (solid line in Fig. 1). The latter appears as a result of the assumed approximation for the Green function given by equation (15). As stated previously (see [10-12]) such an approach applied to mesoscopic QD-based systems, where phonons interact with tunneling carriers allows to include both electron as well as hole contributions in the Green function correlators. Thus, the discussion presented here is in contrast to earlier studies on phonon-assisted AR transmission through hybrid F-QD-S or N-QD-S junctions (see e.g. [2]), where only the electron contribution in the Green function correlators has been taken into account. The numerical analysis presented here also gives rise to a number of new effects. First, splitting of the phonon satellites is observed and it is found that such property does not occur for vanishing intradot Coulomb correlations (see the inset in Fig. 1 for the case of $\left.U^{\prime}=0\right)$. Second, it is seen that phonons interacting with electrons and holes tunneling through the dot give rise to renormalization of the Andreev states. The latter is visible in Figure 1 as narrowing the main elastic resonances with increasing $\lambda$.

The linear conductance in Figure 2 exhibits typical features that were predicted earlier for non-hybrid tunneling junctions of the type $\mathrm{F} / \mathrm{N}-\mathrm{QD}-\mathrm{F} / \mathrm{N}$, namely a suppression of the linear conductance as well as the polaron shift of the conductance peaks, occurring with increasing $\lambda$ (see inset in Fig. 2). As expected, an additional suppression of the conductance for transmission through the F-QD-S hybrid system in the AR regime is induced by the polarization of the $\mathrm{F}$ lead. In contrast to previous results obtained for phonon-assisted transmission through a F-QD-S junction [2], here the linear conductance lacks the phonon satellites. This is consistent with the transport properties reported for the F-QD-F system (see e.g. [11]) described 

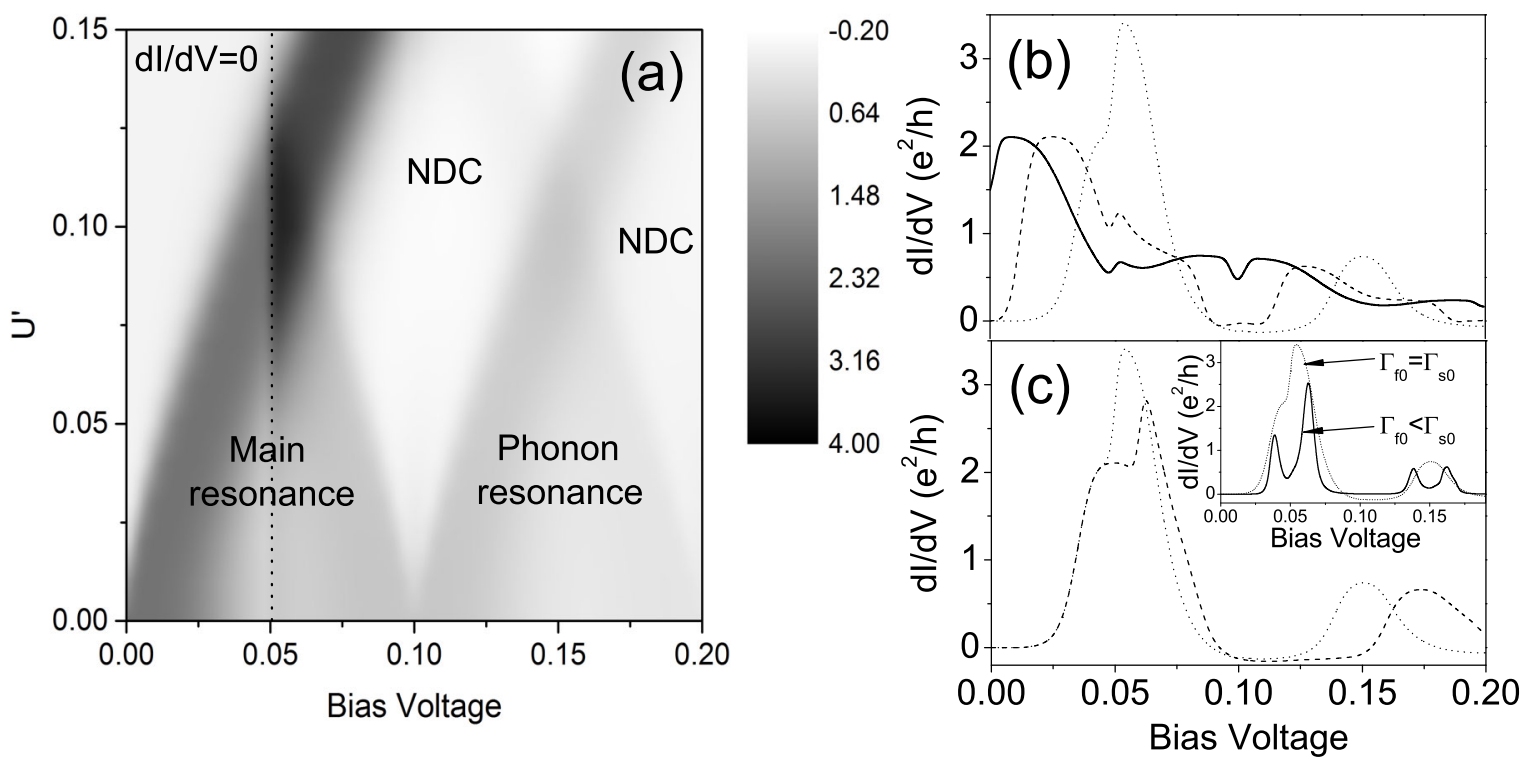

Fig. 3. AR differential conductance, $d I / d V$. The panel (a) shows differential conductance dependent from the bias voltage and for a wide range of the effective Hubbard parameter $U^{\prime}$. The other parameters are $P=0, \varepsilon_{d}^{\prime}+U^{\prime} / 2=0, \Gamma_{f 0}=\Gamma_{s 0}=0.1$ and $\lambda=0.08$. The regions of occurrence of negative differential conductance are indicated as NDC. The dotted line determines values of the differential conductance versus $U^{\prime}$ for the bias voltage fixed at $\Gamma_{s 0} / 2$. The panel (b) shows cross-sections of differential conductance displayed in (a) taken at $U^{\prime}=0.01$ (solid line), $U^{\prime}=0.05$ (dashed line) and $U^{\prime}=0.1$ (dotted line). The panel (c) exhibits differential conductance for two selected electron-phonon couplings: $\lambda=0.08$ (dotted line) and $\lambda=0.096$ (dashed line), both for $U^{\prime}=0.1$. The inset in (c) compares the the AR conductance for $\lambda=0.08$ and $\Gamma_{f 0}=\Gamma_{s 0}=0.1$ (dotted line) to the curve obtained for situation when $\Gamma_{f 0}<\Gamma_{s 0}$ (solid line) with $\Gamma_{f 0}=0.02$ and $\Gamma_{s 0}=0.1$.

by means of the Green function formalism, where the both electron and hole contributions into the Green function correlators have been taken into account.

\subsection{Nonlinear response regime}

Differential conductance for the F-QD-S system in the nonequilibrium situation is displayed in Figure 3. Figure 3a shows the map dependence of the $d I / d V$ quantity on bias voltage as well as on the effective Coulomb intradot repulsion $U^{\prime}$, for the case of the electron-hole symmetry, $\varepsilon_{d}^{\prime}+U^{\prime} / 2=0$. The dark regions appear at bias voltages for which the differential conductance is enhanced. The main maximum corresponds to tunneling through the Andreev levels, whereas the satellite peaks come from transmission through the phonon states (in Fig. 3a only the first phonon maximum is visible). It is known [21] that for vanishing on-dot repulsion and for the system without phonons, the AR transmission occurs through the Andreev levels leading to a variety of conductance properties depending on the relation between the strength of the F-QD and QD-S couplings. In particular, it was shown that for the symmetrical coupling between the dot and the leads (which is the case considered here), $\Gamma_{f 0}=\Gamma_{s 0}$, the overlap of the broadenings of the Andreev levels gave rise to the perfect AR phenomenon with differential conductance being enhanced up to $4 e^{2} / h$. The corresponding result for $U^{\prime}=0$ in Figure 3a shows that phonons may significantly reduce the $d I / d V$ quantity, however for strong enough effective Coulomb correlations on the dot the conductance may be again enhanced to reach even the value of $3.5 e^{2} / h$. The latter property is due to interplay between the strength of the QD-phonon coupling, effective intradot Coulomb repulsion and the broadening of the dot energy levels, which is determined by the relation between $\Gamma_{f 0}$ and $\Gamma_{s 0}$. Besides, it is seen that for a wide range of $U^{\prime}$ the main resonance of the conductance exhibits a step located along the dashed line at bias voltage $\Gamma_{s 0} / 2$ in Figure $3 \mathrm{a}$. The behavior of the side phonon maximum visible in Figure $3 \mathrm{a}$ is similar, i.e. it experiences enhancement with increasing $U^{\prime}$, however the additional step within the conductance peak (analogous to the one occurring along the dashed line at bias voltage $\Gamma_{s 0} / 2$ ) is not observed. Finally, it is found that the effect of negative differential conductance (NDC) may appear between the conductance maxima.

In order to discuss in more detail the transport features of the system shown in Figure 3a, the selected crosssections are taken at $U^{\prime}=0.01, U^{\prime}=0.05$ and $U^{\prime}=0.1$, and displayed in Figure $3 \mathrm{~b}$ as the solid, dashed and dotted lines, respectively. The conductance maximum shifts towards higher transport voltages with increasing $U^{\prime}$ and, as discussed above, finally it may be significantly enhanced when $U^{\prime}=\Gamma_{s 0}$. It is also clearly seen in Figure $3 \mathrm{~b}$ that if $0<U^{\prime}<\Gamma_{s 0} / 2$ then the conductance does not vanish at bias voltages below the threshold $U^{\prime} / 2$ (it is assumed that $\varepsilon_{d}=-U^{\prime} / 2$ at $\left.V=0\right)$. As stated in reference [21], such an effect is due to interference that appears when tunnelling processes occur through the doubly occupied dot and when the broadenings of the Andreev bound states overlap. As expected, if the $\lambda$ parameter increases then 
a suppression of the conductance is observed. The phononinduced suppression of the $d I / d V$ quantity is shown in Figure 3c where the characteristics obtained for $\lambda=0.08$ (dotted line, the same as in Fig. 3b) is compared to the result calculated for $\lambda=0.096$ (dashed line). Simultaneously, note that the position of the additional step in the conductance maxima is $\lambda$-independent, i.e. for the assumed QD-S coupling strength it emerges at bias voltage $\Gamma_{s 0} / 2$. The origin of appearance of this additional step may be explained in terms of relation between the strength of the F-QD and QD-S couplings (see the inset in Fig. 3c). If $\Gamma_{f 0}<\Gamma_{s 0}$, then the maximum of main resonance becomes split into two peaks corresponding to transmission through the Andreev bound states. The minimum between these two peaks in the main elastic resonance determines the position of the steps visible in Figures $3 \mathrm{a}-3 \mathrm{c}$ at bias voltage $\Gamma_{s 0} / 2$. The solid line in the inset in Figure $3 \mathrm{c}$ shows that the phonon satellites may also become split provided that the strength of the F-QD coupling is sufficiently weaker than the strength of the coupling between the dot and the S lead. Finally, the characteristics displayed in Figure 3c reveal that the phonon-induced conductance suppression may be accompanied by widening of the resonance peaks (compare the dotted and dashed line). This is due to the fact that in case of non-vanishing effective intradot Coulomb correlations, the side phonon resonances associated with the dot energy level $\varepsilon_{d}^{\prime}$ may overlap with resonances originating from transmission through the $\varepsilon_{d}^{\prime}+U^{\prime}$ level. The latter is more readily observed for the case of $\Gamma_{f 0}<\Gamma_{s 0}$ (not shown for $\lambda=0.096$ ) rather than for the considered here situation of symmetrical F-QD and QD-S couplings.

From Figure 3a as well as from the inset in Figure 3c it follows that the NDC effect occurs if the condition $\Gamma_{f 0}=\Gamma_{s 0}$ together with $U^{\prime}>0$ is met. When additionally phonon-assisted AR transmission is taken into account, then the phonon resonances give rise to NDC oscillations. Since the spin-polarized AR is considered here, we have found that the mechanism of the NDC phenomenon in F-QD-S systems may also significantly depend on the polarization of the F lead. This is illustrated in Figure 4, where the differential conductance calculated as a function of bias voltage, for a fixed $U^{\prime}$ and for selected polarization parameters $P$ is shown. The insets in Figure 4 exhibit the maps od differential conductance, from which one may readily determine the $P$-dependent limits of occurrence of the NDC effect. If $\lambda=0$ (Fig. 4a) then at $P=0$ the perfect AR is accompanied by the most significant NDC. With increasing $P$ a suppression of the conductance is observed with simultaneous NDC reduction. Finally, NDC effect becomes drastically suppressed in the vicinity of $P=6.5$ (inset in Fig. 4a) thus acquiring almost zero values. In turn, when $\lambda>0$ (Fig. 4b), then the perfect $\mathrm{AR}$ is never met, the quantity $d I / d V$ is even more suppressed, phonon-induced renormalization of the Andreev bound states appears (resonance peaks are narrowed down) and the oscillations of NDC occur. With increasing polarization of the F lead the NDC is almost entirely reduced in the vicinity of $P=5.5$ (inset in Fig. 4b).

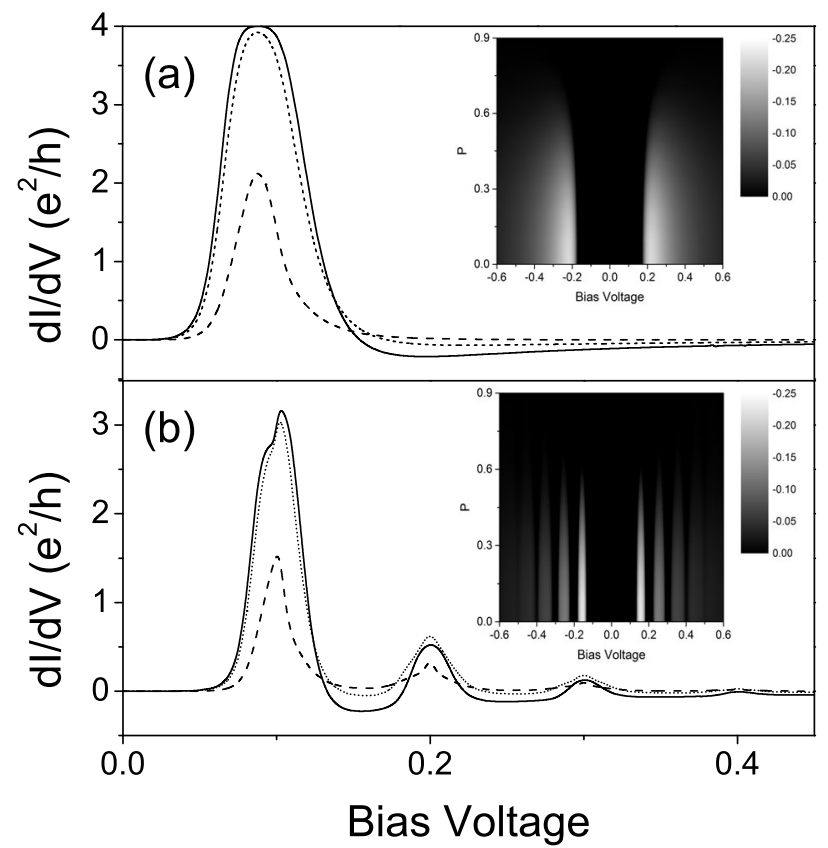

Fig. 4. Differential AR conductance versus bias voltage, calculated for transmission through the system with dot noninteracting with phonons (a), $\lambda=0$, and for the dot interacting with phonons (b), $\lambda=0.08$. Different curves are obtained for selected polarizations of the F lead: $P=0$ (solid lines), $P=0.5$ (dotted lines) and $P=0.9$ (dashed lines). The other parameters are $U^{\prime}=0.2, \Gamma_{f 0}=\Gamma_{s 0}=0.1$ and $\varepsilon_{d}^{\prime}=-U^{\prime} / 2$. The insets show the corresponding map dependencies of the negative differential conductance on the bias voltage and on the $\mathrm{F}$ lead polarization parameter $P$.

\section{Summary and conclusions}

Phonon-assisted tunneling through the hybrid junction based on a single-level, interacting quantum dot, attached to one ferromagnetic and one superconducting leads has been studied theoretically within the non-equilibrium Green function approach. Properties of the spin-polarized transmission through the system have been considered in the Andreev reflection regime. As a result of the approach applied to evaluate the Green function correlators of the system, i.e. by including electron and hole contributions in relevant Green function formulas, we have shown that the side bands due to phonon emission may appear on both sides of the main resonances in the spectral function. The latter is accompanied by phonon-induced renormalization of the Andreev bound states. Moreover, splitting of the phonon satellite resonances due to AR processes is found to appear in case of nonvanishing effective ondot Coulomb correlations. The results obtained for the equilibrium situation prove that the F-QD-S hybrid system exhibits similar behavior as observed earlier for tunnelling through N/F-QD-N/F type junctions [10-12], i.e. such features as the polaron shift, suppression of the linear conductance with increasing on-dot electron-phonon interactions as well as the lack of phonon satellites in the linear conductance may be predicted. 
An analysis of the map dependencies of differential conductance on bias voltage, on a wide range of the effective Coulomb on-dot repulsion parameter $U^{\prime}$ as well as on the the polarization $P$ of the $\mathrm{F}$ lead, has revealed a couple of new transport effects that were not earlier reported in literature. First, it is found that the location, symmetry and height of the main and phonon resonance peaks are determined by interplay between the electron-phonon interactions on the dot, the effective intradot Coulomb correlations and the relation between the couplings of the dot to the external electrodes. The map dependencies show also that the differential conductance may be significantly enhanced even in the presence of the Franck-Condon blockade provided that the Coulomb correlations on the dot are strong enough to satisfy the condition $U^{\prime}=\Gamma_{s 0}$. Moreover, it is predicted that the phononassisted transmission gives rise to renormalization of the Andreev bound states and that the phonon side peaks in the AR conductance may exhibit a splitting into two peaks due to AR processes in nonequilibrium situation if $\Gamma_{f 0}<\Gamma_{s 0}$.

Second, a mechanism leading to the negative differential conductance is discussed. The NDC effect may appear in the hybrid F-QD-S system in the AR regime if the dot with nonvanishing intradot Coulomb correlations is symmetrically coupled to the external electrodes $\left(\Gamma_{f 0}=\Gamma_{s 0}\right)$. Moreover, NDC may be significantly enhanced by manipulating the polarization of the F electrode. Though in general NDC may occur in the system without phonons, it is shown that the polaron transmission gives rise to oscillations of NDC and thus the corresponding maps of oscillating conductance have been calculated for a wide range of bias voltages and polarizations of the ferromagnetic lead.

This work was supported by a 'Iuventus Plus' project No. IP2011 059471 in years 2012-2014 and the National Science Center in Poland as the Project No. DEC-2013/10/E/ ST3/00213.

\section{Appendix: Derivation of the Green functions $G^{r}(\omega)$ and $G^{<}(\omega)$}

In order to derive the formulas given by equations (15) and (16) we start calculations with the definitions of the time-dependent Green functions $\mathbf{G}^{<}\left(t, t^{\prime}\right)$ and $\mathbf{G}^{r}\left(t, t^{\prime}\right)$ taken at $t^{\prime}=0$. In particular, for $\mathbf{G}^{<}(t, 0)$ one gets:

$$
\begin{aligned}
\mathbf{G}^{<}(t, 0) & =i\left\langle\tilde{\boldsymbol{\Psi}}^{\dagger} \otimes e^{i \tilde{H} t} \tilde{\boldsymbol{\Psi}} e^{-i \tilde{H} t}\right\rangle \\
= & i\left\langle\left(\boldsymbol{\Psi}^{\dagger} \circ \boldsymbol{\Omega}^{\dagger}\right) \otimes e^{i\left(\tilde{H}_{e l}+H_{p h}\right) t}(\boldsymbol{\Psi} \circ \boldsymbol{\Omega}) e^{-i\left(\tilde{H}_{e l}+H_{p h}\right) t}\right\rangle,
\end{aligned}
$$

where $\tilde{H}_{e l}=H_{f}+H_{s}+\tilde{H}_{d}+\tilde{H}_{t}$ denote the electron part of the transformed Hamiltonian (12). Using the BakerCampbell-Hausdorff formula satisfied by any pair of $\mathbf{A}$ and $\mathbf{B}$ operators [13],

$$
\begin{aligned}
e^{A} B e^{-A}= & B+\frac{1}{1 !}[A, B]+\frac{1}{2 !}[A,[A, B]] \\
& +\frac{1}{3 !}[A,[A,[A, B]]]+\ldots,
\end{aligned}
$$

taking into account that $\left[H_{p h}, \boldsymbol{\Psi}\right]=0$ and $\left[\tilde{H}_{e l}, \boldsymbol{\Omega}\right]=0$, and using the relationship $\tilde{\boldsymbol{\Psi}}^{\dagger} \otimes \tilde{\boldsymbol{\Psi}}=\left(\boldsymbol{\Psi}^{\dagger} \circ \boldsymbol{\Omega}^{\dagger}\right) \otimes(\boldsymbol{\Psi} \circ \boldsymbol{\Omega})=$ $\left(\boldsymbol{\Psi}^{\dagger} \otimes \boldsymbol{\Psi}\right) \circ\left(\boldsymbol{\Omega}^{\dagger} \otimes \boldsymbol{\Omega}\right)$, finally we obtain

$$
\begin{aligned}
\mathbf{G}^{<} & (t, 0) \\
& =i\left\langle\left(\boldsymbol{\Psi}^{\dagger} \circ \boldsymbol{\Omega}^{\dagger}\right) \otimes\left[\left(e^{i \tilde{H}_{e l} t} \boldsymbol{\Psi} e^{-i \tilde{H}_{e l} t}\right) \circ\left(e^{i H_{p h} t} \boldsymbol{\Omega} e^{-i H_{p h} t}\right)\right]\right\rangle \\
& =i\left\langle\boldsymbol{\Psi}^{\dagger} \otimes e^{i \tilde{H}_{e l} t} \boldsymbol{\Psi} e^{-i \tilde{H}_{e l} t}\right\rangle \circ\left\langle\boldsymbol{\Omega}^{\dagger} \otimes e^{i H_{p h} t} \boldsymbol{\Omega} e^{-i H_{p h} t}\right\rangle \\
& =i\left\langle\boldsymbol{\Psi}^{\dagger}(0) \otimes \boldsymbol{\Psi}(t)\right\rangle_{\tilde{H}_{e l}} \circ\left\langle\boldsymbol{\Omega}^{\dagger}(0) \otimes \boldsymbol{\Omega}(t)\right\rangle_{H_{p h}}, \quad \text { (A.3) }
\end{aligned}
$$

where the matrix $\left\langle\boldsymbol{\Omega}^{\dagger}(0) \otimes \boldsymbol{\Omega}(t)\right\rangle_{H_{p h}}$ reads

$$
\left\langle\boldsymbol{\Omega}^{\dagger}(0) \otimes \boldsymbol{\Omega}(t)\right\rangle_{H_{p h}}=\left(\begin{array}{llll}
a & b & a & b \\
c & d & c & d \\
a & b & a & b \\
c & d & c & d
\end{array}\right),
$$

with $a \equiv\left\langle X^{\dagger}(0) X(t)\right\rangle, b \equiv\langle X(0) X(t)\rangle, c \equiv\left\langle X^{\dagger}(0) X^{\dagger}(t)\right\rangle$ and $d \equiv\left\langle X(0) X^{\dagger}(t)\right\rangle$. In a similar manner for the retarded Green function one gets

$$
\begin{aligned}
\mathbf{G}^{r}(t, 0)= & \tilde{\mathbf{G}}^{r}(t, 0) \circ\left\langle\boldsymbol{\Omega}(t) \otimes \boldsymbol{\Omega}^{\dagger}(0)\right\rangle_{H_{p h}} \\
& +\theta(t) \tilde{\mathbf{G}}^{<}(t, 0) \circ\left\{\left\langle\boldsymbol{\Omega}(t) \otimes \boldsymbol{\Omega}^{\dagger}(0)\right\rangle_{H_{p h}}\right. \\
& \left.-\left\langle\boldsymbol{\Omega}^{\dagger}(0) \otimes \boldsymbol{\Omega}(t)\right\rangle_{H_{p h}}\right\} .
\end{aligned}
$$

Assuming that phonons have the same energy $\omega_{0}$, the elements of the matrices $\left\langle\boldsymbol{\Omega}^{\dagger}(0) \otimes \boldsymbol{\Omega}(t)\right\rangle_{H_{p h}}$ and $\left\langle\boldsymbol{\Omega}(t) \otimes \boldsymbol{\Omega}^{\dagger}(0)\right\rangle_{H_{p h}}$ become [13]:

$$
\begin{aligned}
\left\langle X(0) X^{\dagger}(t)\right\rangle & =\left\langle X^{\dagger}(0) X(t)\right\rangle=\sum_{n=-\infty}^{\infty} L_{n} e^{i n \omega_{0} t}, \text { (A.6) } \\
\left\langle X(t) X^{\dagger}(0)\right\rangle & =\left\langle X^{\dagger}(t) X(0)\right\rangle=\sum_{n=-\infty}^{\infty} L_{n} e^{-i n \omega_{0} t} \\
\langle X(0) X(t)\rangle & =\left\langle X^{\dagger}(0) X^{\dagger}(t)\right\rangle=\sum_{n=-\infty}^{\infty}(-1)^{n} L_{n} e^{i n \omega_{0} t}, \\
\langle X(t) X(0)\rangle & =\left\langle X^{\dagger}(t) X^{\dagger}(0)\right\rangle=\sum_{n=-\infty}^{\infty}(-1)^{n} L_{n} e^{-i n \omega_{0} t}
\end{aligned}
$$

where the coefficient $L_{n}$ is defined in equation (18). Thus,

$$
\begin{aligned}
& \left\langle\boldsymbol{\Omega}^{\dagger}(0) \otimes \boldsymbol{\Omega}(t)\right\rangle_{H_{p h}}=\sum_{n=-\infty}^{\infty} L_{n} e^{i n \omega_{0} t} \mathbf{M}_{n}, \\
& \left\langle\boldsymbol{\Omega}(t) \otimes \boldsymbol{\Omega}^{\dagger}(0)\right\rangle_{H_{p h}}=\sum_{n=-\infty}^{\infty} L_{n} e^{-i n \omega_{0} t} \mathbf{M}_{n},
\end{aligned}
$$


with matrix $\mathbf{M}_{n}$ given by equation (17). Finally, after substituting (53) and (54) into (46) and (48) and taking the Fourier transforms one arrives at the needed expressions for the Green functions $\mathbf{G}^{r}(\omega)$ and $\mathbf{G}^{<}(\omega)$.

\section{References}

1. A.F. Andreev, J. Exp. Theor. Phys. 19, 1228 (1964)

2. P. Zhang, Y.-X. Li, J. Phys.: Condens. Matter 21, 095602 (2009)

3. L. Bai, Z.-Z. Zhang, L. Jiang, Phys. Lett. A 375, 661 (2011)

4. S.-N. Zhang, W. Pei, T.-F. Fang, Q.-F. Sun, Phys. Rev. B 86, $104513(2012)$

5. B.H. Wu, J.C. Cao, C. Timm, Phys. Rev. B 86, 035406 (2012)

6. Q. Chen, Y.-H. Deng, Commun. Theor. Phys. 56, 517 (2011)

7. K. Bocian, W. Rudziński, Acta Physica Polonica A $\mathbf{1 2 6}$ $374(2014)$

8. P. Szabó, P. Samuely, J. Kačmarčik, A.G.M. Jansen, T. Klein, J. Marcus, C. Marcenat, Supercond. Sci. Technol. 16, $162(2003)$

9. E.C. Siqueira, G.G. Cabrera, Phys. Rev. B 81, 094526 (2010)

10. M. Galperin, A. Nitzan, M.A. Ratner, Phys. Rev. B 73, 045314 (2006)
11. R.-Q. Wang, Y.-Q. Zhou, B. Wang, D.Y. Xing, Phys. Rev. B 75, 045318 (2007)

12. W. Rudziński, J. Phys.: Condens. Matter 20, 275214 (2008)

13. G.D. Mahan, Many-Particle Physics (Plenum Press, New York, 2000)

14. K. Flensberg, Phys. Rev. B 68, 205323 (2003)

15. Z.-Z. Chen, R. Lü, B.-F. Zhu, Phys. Rev. B 71, 165324 (2005)

16. Z.Y. Zeng, Baowen Li, F. Claro, Phys. Rev. B 68, 115319 (2003)

17. D.N. Zubarev, Sov. Phys. Usp. 3, 320 (1960)

18. X. Cao, Y. Shi, X. Song, S. Zhou, H. Chen, Phys. Rev. B 70, 235341 (2004)

19. N.S. Wingreen, K.W. Jacobsen, J.W. Wilkins, Phys. Rev. B 40, 11834 (1989)

20. A.-P. Jauho, N.S. Wingreen, Y. Meir, Phys. Rev. B 50, 5528 (1994)

21. K. Bocian, W. Rudziński, Eur. Phys. J. B 86, 439 (2013)

Open Access This is an open access article distributed under the terms of the Creative Commons Attribution License (http://creativecommons.org/licenses/by/4.0), which permits unrestricted use, distribution, and reproduction in any medium, provided the original work is properly cited. 